\title{
STRESS PRE-ENTRY IN MOTOR SYSTEMS
}

\author{
Donald G. MacKay \\ University of California, Los Angeles
}

\begin{abstract}
Stress pre-entry is the occurrence, before it is due, of the stressed component in a series of rapidly produced movements. The phenomenon appeared in speech (experimentally produced spoonerisms) and in patterns of finger movement: serial-order errors were usually a stressed element entering before its time. The probability of serial-order errors was also found to increase as a function of rate of action. Several explanations are possible, but the model that best fits the data is one of a scanning device for determining serial order of rapidly produced behavior.
\end{abstract}

Errors in the serial order of action are crucial to theories of behavior in the same sense that illusions are crucial to theories of perception. But although the problem of serial order in behavior has attracted considerable theoretical attention (Milner, 1961; Conrad, 1965; Bryden, 1967; Wickelgren, 1969) since Lashley's famous paper (1961), few experiments on errors in serial order have appeared (MacNeilage, 1964; Shaffer and Hardwick, 1968, 1969). The experiments reported here focus on a limited aspect of the problem, namely, the effects of motor intensity (or stress) and rate of action on serial reversals in speech and in patterns of finger movement.

We began by examining the role of stress in spoonerisms (involuntary reversals in the serial order of phonemes in natural or conversational speech). Next we found a method of experimentally inducing spoonerisms that allowed a systematic test of the effects of stress and rate on serial reversals in speech. We then carried out an analogous experiment on serial-order errors in patterns of finger movement, our question being whether stress and rate play a similar role in motor systems other than speech. Finally we developed a 
model to account for the effects of stress and rate on serial-order reversals in these two motor systems.

\section{SPOONERISMS IN GERMAN}

Our interest in the effect of stress in serial-order errors began with an examination of 124 spoonerisms in German (Meringer and Mayer, 1895; Meringer, 1908). Our reasons for considering this corpus were, first, its size and, second, Meringer's care in collecting the data (see MacKay, 1970). It appeared that one of the reversed phonemes in these spoonerisms often originated in a stressed syllable and the other in an unstressed one. Moreover, the stressed element usually seemed to be the one that occurred before it was due, as in

$$
\begin{aligned}
& \text { vaga BUND } \rightarrow \text { vabagund, or } \\
& \text { ge BRAUCH } \rightarrow \text { begrauch, }
\end{aligned}
$$

where the intruding $\mathrm{B}$ in both examples originated in a stressed syllable (capitalized; the arrow means 'was spoken as'). Our first question was whether this apparent effect of stress in spoonerisms exceeded chance expectation.

Our analyses were carried out within the following definitional framework. Within-word spoonerisms were defined as reversals of phonemes within a single word, as in Examples 1 and 2. Spoonerisms with reversed phonemes originating in different words were not included in this analysis. ${ }^{1}$ An intruding phoneme was defined as the phoneme executed before its time, as B in Examples 1 and 2. A lagging phoneme was defined as the phoneme executed after its time, as $\mathrm{G}$ in Examples 1 and 2. The intruding and lagging phonemes originated in different syllables in all but one of the examples in Meringer's corpus. Since one syllable is more stressed than all the others in multisyllabic words in German, ${ }^{2}$ syllabic stress was represented as 0 or 1, determined from a standard German dictionary (Wahrig, 1966). An instance of stress pre-entry was counted whenever the intruding pheneme in a spoonerism originated in a stressed syllable. Thus Example 3 represents an instance of stress pre-entry whereas Example 4 does not. Note that only one syllable was counted as stressed:

be GA bung $\rightarrow$ gebabung (example from Meringer)

be GA bung $\rightarrow$ bugabeng (hypothetical example) 
Thus in each word, stress pre-entry implies that the lagging phoneme originated in an unstressed syllable. An instance of stress postentry was counted when the intruding phoneme originated in an unstressed syllable and the lagging phoneme in a stressed one. Example 5 thus represents an instance of stress post-entry:

$$
\text { be GA bung } \rightarrow \text { bebagung (hypothetical example) }
$$

The last definition is of corpus of natural speech: this corpus, consisting of 230 correctly produced sentences from Meringer (comparable to those containing the spoonerisms), was used to determine the null hypotheses.

Two hypotheses guided our analyses. The like-with-like hypothesis was first proposed by Boomer and Laver (1968): "The origin syllable and the target syllable of a slip are metrically similar in that both are salient or both are weak." Using our definition of stress (or salience), this hypothesis predicts that both syllables in withinword spoonerisms should be unstressed with greater than chance probability. Moreover, stress pre-entry should be no more frequent than stress post-entry under this hypothesis. The stress pre-entry hypothesis, however, predicts that stress pre-entry should exceed chance expectation. That is, one of the reversed phonemes should be stressed with greater than chance probability, and stress preentry should be significantly more frequent than stress post-entry.

According to our analyses of Meringer's data, both syllables were unstressed in $18 \%$ of the German spoonerisms, whereas $26 \%$ would be expected by chance (based on the relative frequency of stressed and unstressed syllables in the corpus of natural speech). This difference was not statistically reliable at the .05 level using a chisquare test on the raw data.

However, stress pre-entry was significantly more frequent than chance expectation. Here chance expectation reflected the frequency with which stressed syllables followed unstressed syllables in the multisyllabic words in the corpus of natural speech-which was $23 \%$ of the time. But stress pre-entry appeared $71 \%$ of the time in the corpus of spoonerisms, a difference significant from chance at the .01 level, chi-square test. The same test showed that stress post-entry was significantly less frequent $(11 \%)$ than chance expectation $(51 \%$, based on the frequency with which stressed syllables preceded unstressed ones in the multisyllabic words in the corpus of natural speech). Both findings strongly support the stress pre-entry hypothesis. $^{3}$ 


\section{SPOONERISMS IN ENGLISH}

Is stress pre-entry a universal tendency common to all speakers regardless of language? As a preliminary test of this hypothesis, we analyzed an American corpus of 55 spoonerisms published by Bawden (1900; see MacKay, 1970), apparently without knowledge of Meringer's work. Stress was tabulated for within-word spoonerisms as before, with the results below. Since we lacked a corpus of correctly produced sentences from Bawden's speakers, we based the null hypothesis on a corpus of 255 multisyllabic words randomly sampled from the index at the top of odd-numbered pages of a large American dictionary.

According to our analysis of Bawden's data, stress pre-entry in the English spoonerisms (57\%) was more common than chance expectation ( $11 \%$, calculated as before); using a chi-square test, a difference significant at the .01 level. Similarly, stress post-entry was less common $(28 \%)$ than chance expectation $(55 \%)$, a difference significant at the .01 level, same test. Both findings support a stress pre-entry hypothesis for within-word spoonerisms in English.4

Both syllables were unstressed in the English spoonerisms only $15 \%$ of the time, whereas $34 \%$ would be expected by chance. Combining these data with those from the German spoonerisms, we found that both syllables were unstressed significantly less frequently than chance expectation at the .02 level, chi-square test. This finding directly contradicts the like-with-like hypothesis of Boomer and Laver (1968). The contradiction may be due to the fact that Boomer and Laver based their hypothesis on a conglomeration of errors that included substitutions, anticipations, perseverations, and omissions. But since the role of stress varies in different classes of errors (see MacKay, 1969), lumping different classes of errors together seems unwise. The like-with-like hypothesis simply does not hold for syllabic stress in within-word spoonerisms, although it may hold for other errors, given appropriate statistical analyses.

\section{EXPERIMENTALLY INDUCED SPOONERISMS}

We discovered that spoonerisms sometimes occur when subjects rapidly repeat a sequence of nonsense syllables such as 'TAY GAY BAY DAY.' In the experiment to be described, we systematically varied which syllable in this sequence was stressed. Under the stress 
pre-entry hypothesis we predicted that when spoonerisms did occur, one of the reversed components would be stressed with greater than chance expectation. Moreover, stress pre-entry in these experimentally induced spoonerisms should be more frequent than stress post-entry.

This experiment offered several advantages over our analyses of spoonerisms in natural speech. First, by using nonsense materials we could effectively rule out the semantic or 'deep' interpretations that have dominated the discussion of speech errors since Freud. Second, we could experimentally manipulate speech rate, a factor that went uncontrolled in observations of natural speech. Third, we could test the informational hypothesis of stress pre-entry. This hypothesis maintains that in natural speech, stressed syllables and words are more informative (in the information-theory sense) than unstressed ones, so that this may be why they enter before their time, and not because of motor intensity per se. By systematically varying which syllable was stressed we were able to determine whether motor intensity causes stress pre-entry independent of information value.

\section{Method}

-Subjects and apparatus-Twenty-two right-handed students at Edinburgh University were paid for their participation in the experiment. Their responses were recorded with a Revox tape recorder, and their errors were scored later from the tape.

-Experimental design-Two factors were varied, rate of speech and which syllable in the sequence was stressed.5 Each subject was instructed to speak at two rates for each stress condition: maximum rate and normal rate. The order of the two rates was counterbalanced across subjects and stress conditions.

There were five stress conditions. In the no-stress condition, the subjects were instructed not to emphasize any of the syllables. In the remaining four conditions, one of the syllables was emphasized (made louder than the others), giving Examples 6 to 9, stressed syllables capitalized. This particular sequence 
of syllables was chosen to maximize the probability of serial-order errors. ${ }^{6}$ But note that our hypothesis depends on whether an element in the sequence was stressed or unstressed, a systematically varied factor that was independent of the particular sequence chosen.

In a practice session before the experiment, the subject repeated each stress pattern five times at both rates of speech. In the experiment proper, he was instructed to continue repeating the sequence until told to stop. For both rate conditions, this stop signal came after 20 repetitions of the sequence. Order of the stress conditions was randomized across subjects.

Errors were scored in a pattern only when the first syllable was produced correctly.

\section{Results}

Spoonerisms were defined as before. For example, 'TAY bay gay day' represents a spoonerism, whereas 'TAY bay bay day' does not. The probability of spoonerism per sequence of four syllables was .015 (averaged over stress and rate conditions). As might be expected, the maximal rate (.280 sec per syllable) was considerably faster than the normal rate (.358 sec per syllable). As to frequency of spoonerisms for the two rates, $57 \%$ of the spoonerisms occurred at maximal rate and $43 \%$ at normal rate, a difference reliable at the .05 level, chi-square test. The importance of this effect of rate for theories of spoonerisms is outlined in the discussion.

Of the spoonerisms 95\% occurred between adjacent syllables in the sequence. Moreover, one of the reversed components was stressed much more often than chance expectation. Only $40 \%$ of the reversals should involve a stressed component by chance, where chance reflects the frequency of stressed and unstressed elements in all of the experimental conditions. But in fact, $51 \%$ of the reversals involved a stressed component (see Table 1), which exceeds chance expectation at the .05 level, chi-square test.

Table 1. Experimentally induced spoonerisms as a function of stress

\begin{tabular}{lcccc}
\hline & $\begin{array}{c}\text { One } \\
\text { syllable } \\
\text { stressed }\end{array}$ & $\begin{array}{c}\text { Neither } \\
\text { syllable } \\
\text { stressed }\end{array}$ & $\begin{array}{c}\text { Stress } \\
\text { pre-entry }\end{array}$ & $\begin{array}{c}\text { Stress } \\
\text { post-entry }\end{array}$ \\
\hline $\begin{array}{c}\text { Reversals } \\
\text { (percent) }\end{array}$ & 51 & 49 & 90 & 10 \\
$\begin{array}{c}\text { Chance } \\
\text { (percent) }\end{array}$ & 40 & 60 & 50 & 50 \\
\hline
\end{tabular}


Moreover, the experimental spoonerisms obeyed a stress pre-entry rule: the stressed element entered before it was due much more frequently than after. These data are shown in Table 1 , where it can be seen that stress pre-entry was much more frequent than chance expectation, and stress post-entry much less frequent than chance, a statistically reliable finding at the .01 level, chi-square test.

\section{SERIAL ORDER IN PATTERNS OF FINGER MOVEMENT}

Is stress pre-entry a universal error tendency common to all motor systems or does it only appear in speech? As a first step in answering this question, we examined serial-order errors in patterns of finger movement. The experiment was analogous to that reported above for speech: the subjects rapidly repeated a sequence of finger taps on telegraph keys. As before, the stress pre-entry hypothesis was tested, here by systematically varying which finger tap was emphasized. Our prediction was that, as in speech, stressed movements would occur before their time more frequently than chance expectation.

\section{Method}

-Subjects-The subjects were the same 22 students who had participated in the experiment just reported, the order of the two experiments being counterbalanced across subjects.

-Apparatus-The main piece of apparatus was a four-channel Gerbrands event recorder, which recorded the time and duration of the responses. A response was a $1-\mathrm{mm}$ depression of a standard telegraph key. There were four keys in all, one for each finger of the left hand. By placing his left arm on the table, the subject could easily rest each finger on one of the keys, which were mounted on a piece of bristol board attached to the table.

-Experimental design-The movement pattern in all experimental conditions was exactly the same. If the fingers of the left hand are labeled $a$ to $d$ from little finger to index finger, then the pattern was $a c b d$. It might be argued that this particular sequence encourages the serial error $a b c d$. This may be true, but our question was whether the abcd errors became more frequent when $b$ was stressed than when it was not.

Again, the independent variables were the rate of executing the sequence and the stress pattern. For each stress condition the subject ran through the sequence at two rates: as fast as he could, and at a normal rate. Order of the two rates was counterbalanced across subjects and stress conditions. As before, there were five stress conditions: a different finger was stressed or emphasized 
in four of the stress conditions, and in the fifth, none of the fingers was stressed. Order of the stress conditions was randomized across subjects. In the actual experiment, the subject repeated each movement pattern 20 times for each rate and stress condition (following a practice session in which he produced each stress pattern five times at both rates).

As before, errors were scored only when the first element was produced correctly.

\section{Results}

The probability of reversal was .01 per movement pattern, averaged over stress and rate conditions. As might be expected, the actual rate of movement (in seconds per key press) diminished with maximal-rate instructions: the response rate was .119 for the slow rate and .088 for the maximal rate. And more serial-order errors occurred at the maximal rate $(60 \%)$ than at the slow rate $(40 \%)$, a finding significant at the .05 level, chi-square test. For some reason, serial reversals became more likely with faster rates of movement.

Again, the actions reversed were usually temporally adjacent in the sequence, and they frequently involved a stressed component. As can be seen in Table 2, one of the movements was stressed in $53 \%$ of the reversals, significantly more than the $40 \%$ expected by chance (.05 level, chi-square test). Also shown in Table 2 is the prob-

Table 2. Serial-order errors in patterns of finger movements as a function of stress

\begin{tabular}{lcccc}
\hline & $\begin{array}{c}\text { One tap } \\
\text { stressed }\end{array}$ & $\begin{array}{c}\text { Both taps } \\
\text { unstressed }\end{array}$ & $\begin{array}{c}\text { Stress } \\
\text { pre-entry }\end{array}$ & $\begin{array}{c}\text { Stress } \\
\text { post-entry }\end{array}$ \\
\hline $\begin{array}{c}\text { Reversals } \\
\text { (percent) }\end{array}$ & 53 & 47 & 86 & 14 \\
$\begin{array}{c}\text { Chance } \\
\text { (percent) }\end{array}$ & 40 & 60 & 50 & 50 \\
\hline
\end{tabular}

ability of stress pre-entry and post-entry (defined as for speech). Stress pre-entry was significantly more frequently than chance expectation at the .01 level, chi-square test. Similarly, stress postentry was less frequent than chance expectation (.01 level, same test). As in spoonerisms, errors in the serial order of finger movements obeyed a law of prior entry, with stressed elements occurring before their time. 
-Controls-Two of the preceding conditions were rerun on 11 righthanded students at UCLA. Conditions $a c B d$ (stress on finger $B$ ) and $a c b d$ (no stress) were produced 20 times at maximal rate (with practice trials as before). Then four control conditions were added to test the hypotheses outlined below, the order of all conditions being counterbalanced across subjects.

The von Restorff hypothesis was one of those tested. Since only one element was stressed in the critical conditions of the main experiment, we wondered whether this element, being different from the others, stood out and was therefore more prone to serial-order reversal. To test this, we ran a condition where all the finger taps but one were stressed. The finger pattern was $A C b D$, stressed fingers capitalized. Under the von Restorff hypothesis we predicted that the unstressed element in $A C b D$ would occur before its time as often as the stressed element in $a c B d$. However, the data showed that stress pre-entry in $a c B d$ was much more common than unstressed pre-entry in $A C b D$. Finger $b$ occurred before its time more frequently when stressed than when unstressed, a difference significant at the .05 level, chi-square test. This finding conclusively rules out an explanation of stress pre-entry as a von Restorff effect.

The second hypothesis tested in the control conditions was the relational hypothesis. The reason for stress pre-entry may lie in the relation between stressed and unstressed elements, the stressed elements entering before unstressed elements but not before other stressed elements. To test this, we ran a control condition in which all of the elements were stressed. The data showed that serial-order reversals were no more common when all of the elements were stressed $(N=5)$ than when all were unstressed $(N=5)$, which supports the relational hypothesis. Moreover pre-entry of finger $b$ was more frequent in condition $a c B d$ than in condition $A C B D$, although this difference just missed statistical reliability at the .05 level using a chi-square test. Nevertheless, these data tentatively support the relational hypothesis, since stressed elements tended to advance on unstressed elements but not on other stressed ones.

The last hypothesis tested here was the specificity hypothesis. In order to rule out the possibility that our results were specific to the left hand, we ran condition $a c B d$ using the right hand of our righthanded subjects. Serial-order errors were much less frequent with the right hand, suggesting a dexterity effect. Nevertheless a stress pre-entry trend was observed in the right hand, although it failed statistical significance because of the small number of errors. 
Finally, the pattern $d b C A$ was run with the left hand to test the generality of the stress pre-entry phenomenon. Stress pre-entry occurred with about the same frequency in $d b C a$ as in $a c B d$, indicating that stress pre-entry is not tied to a particular movement pattern.

\section{A MODEL OF STRESS PRE-ENTRY}

Serial-order reversals in sensory or input systems bear a curious resemblance to stress pre-entry in motor or output systems. When two visual stimuli of similar form are presented in succession, a dim one first and a very intense one second, the second is sometimes perceived as preceding the first. The probability of this reversal in the serial order of perception can be expressed as

$$
P(R)=K(b-a),
$$

where $P(R)$ is the probability of reversal, $K$ is a constant, and $a$ and $b$ are the intensities of the first and second stimuli respectively (Korte, 1915). In this last section, we explore the possibility that motor reversals can be explained with an analogous formula.

There are three rather well documented premises that can be used to explain stress pre-entry in sensory systems:

Premise 1: Excitability of the set of analyzers for a form must be boosted to threshold before the form is perceived (after Sutherland, 1959, and others).

Premise 2: A strong or intense stimulus boosts these analyzers to threshold faster than a weak one. This assumption is backed by both psychophysical and physiological evidence (Pulfrich, 1922; Liang and Piéron, 1942; Lit, 1949; Wilson and Anstis, 1969; Adrian and Matthews, 1927).

Premise 3: The mechanism for judging the serial order of input is independent of the analyzers themselves, in the sense that the rate of activation of the analyzers is not taken into consideration in judgments of serial order.

Although these premises do not encompass all aspects of perception, they suffice to explain perceptual reversals, since a strong stimulus reaching analyzer threshold before a weak one is judged to precede the weak one, even though the weak one preceded in the external world. 
The analogous premises for explaining stress pre-entry in motor systems might then take the form:

Premise 1A: Excitability of a set of motor units for an action must be boosted to threshold for the action to be produced.

Premise 2A: Motor units for stressed actions are boosted to threshold faster than those for unstressed actions.

Premise 3A: The mechanism for sequencing behavior is independent of the motor units themselves (as Lashley, 1961, suggested).

Two objections to this analogy between sensory and motor reversals might be raised. One is that perceptual reversals only occur when visual stimuli are separated by $100 \mathrm{msec}$ or less (Wilson and Anstis, 1969), whereas more than $100 \mathrm{msec}$ usually separate the reversed components in speech. However, it is highly likely that motor reversals originate at a central rather than peripheral levelfor example, in higher-level instructions to the motor units rather than in the motor units themselves. If the rate at which these instructions are given at this central level exceeds the speed at which the final output is produced, the objection is overcome. The objection also becomes irrelevant if time constants in motor units are longer than in perceptual analyzers even though similar principles operate.

A second objection to the analogy might be that stressed output differs from unstressed output in other ways besides intensity. Stressed actions last longer than unstressed ones, and they involve faster rates of movement. However, these concomitants of stress can be predicted, as we will see as we develop our premises into a general model for motor systems.

To develop that model, let us first consider the possibility that sequences of acts to be rapidly performed are represented or displayed in abstract form and proper order in an output buffer, such that the elements in the buffer prime or partially activate corresponding motor units. Independent justification for the assumption of priming or subthreshold activation of motor units prior to final motor output is found in such other errors in speech as anticipations (Lashley, 1961). If this priming varies in direct proportion to stress, then stressed units are closer to threshold (prior to final output) than are unstressed units. ${ }^{7}$ Some justification for this assumption is found in omissions of speech sounds: the sounds omitted are usually unstressed, rather than stressed (Heffner, 1964). 
Let us next consider the possibility that a scanning mechanism determines the serial order of behavior by sweeping over the buffer system in undirectional fashion. By boosting the excitability of the buffer elements, this scanning device eventually triggers the motor units, but at the same time is unconnected to or independent of the motor units themselves (Premise 3A). The scanner, it is proposed, provides a linear boost in excitability of elements in the buffer for a period determined by the rate of scanning. Voluntary control of the rate of scanning varies the rate of action within limits, the upper limit being determined by the duration of temporal summation needed to activate the motor units. If stressed units are already closer to threshold than unstressed units, this summation time is less for boosting stressed units to threshold than unstressed ones (Premise 2A). This postulate allows a precise formulation of the probability of stress pre-entry $[P(S)]$. If $A$ and $B$ are units in serial order in the buffer system with summation times $a$ and $b$ respectively (i.e., $a$ and $b$ represent the time following onset of scanning needed to boost the motor units for $A$ and $B$ to threshold), then

$$
P(S)=K(a-b-c),
$$

where $K$ is a constant and $c$ is the time between onset of scanning $A$ and $B$. Consequently,

$$
\begin{array}{ll}
P(S)=0 & \text { if } a-b-c>0 \\
& \text { or } a<b+c, \text { and } \\
P(S)>0 & \text { if } a-b-c<0 \\
& \text { or } a>b+c .
\end{array}
$$

These formulas derived from the model can be seen to summarize all of our findings. Note that $P(S)$ increases as $c$ becomes smaller. One of the factors influencing $c$ is the separation of the relevant units in the buffer system. All other factors being equal, serial-order reversals between elements in close proximity are to be expected. Thus the fact that reversed phonemes occur closer together in natural speech than would be expected by chance (see MacKay, 1970; Cohen, 1967) is represented in these formulas. Similarly, the fact that reversed actions were usually temporally adjacent in our experiments is captured in Formula 13.

Another factor that determines $c$ (the time between onset of scanning the relevant units in the buffer) is the rate of scanning or, equivalently, the rate of action. With faster rates of scanning, $c$ be- 
comes smaller and $P(S)$ becomes greater in Formula 13, which captures the fact that serial-order reversals became more likely with rapid rates of speech and finger movement in our experiments. Finally, these formulas summarize both the stress pre-entry phenomenon itself and the relational hypothesis by predicting a high frequency of serial-order reversals when $a$ is large and $b$ is small-that is, when the first element is unstressed and the second is stressed, as is usually the case in motor reversals.

Thus developed, the model also explains why stressed speech sounds are longer than unstressed ones (Heffner, 1964). If the motor units for stressed segments reach threshold sooner than those for unstressed ones, then, all other factors being equal, stressed sounds in the final output are bound to be longer than unstressed ones. And finally, the model explains why stressed actions are carried out with faster rates of action than unstressed ones (O'Hala, 1969). The rate of muscular contraction is determined by the number of impulses per second from the motor units (Buller, Eccles, and Eccles, 1960). If the activity of the motor units prior to actual movement is greater for stressed than unstressed actions, then following scanning in this model the final activity of stressed units is also greater, leading to faster rates of movement.

Several limitations of the model should be emphasized. First, it applies to only one aspect of serial-order reversals; many other factors known to influence errors in serial order have been ignored for the sake of simplicity. Second, it is based on a restricted type of behavior; we have no grounds for generalizing our model to the natural behavior patterns and rapidly executed thought sequences that originally sparked Freud's interest in faulty actions. Perhaps Freud was partially right in contending that for natural speech "even the apparently simple cases of speech blunder will be traced to a disturbance caused by a half-repressed idea outside of the intended context" (1914, p. 66). However, Wundt's hypothesis (1907) that these ongoing mental activities often act as distractors (i.e., as catalysts rather than causes of errors) warrants serious investigation as an alternative hypothesis (see n. 5).

\section{Other theories}

Some aspects of scanning models are necessarily vague at this point, partly because the nature of the output buffer is as yet un- 
known. The scanning mechanism developed in the model above is essentially analogous to certain features of a television receiver. But there could be a different scanning mechanism, one based on the principle of the tape-recorder head, which 'reads out' the electromagnetic pattern on a tape. Instead of energizing elements in an interim storage system and thereby activating the motor components, this scanner would serially read out from the buffer a set of values for the motor units. This 'read out' scanner can be made to predict the same findings as the 'receiver' scanner in the model above, and at present I see no way of experimentally distinguishing the two.

Another possibility is that the scanner may release from inhibition the programs in the output buffer rather than activate them directly. This view is related to Lashley's hypothesis that "it is as if [an] aggregate of words were in a state of partial excitation, held in check by the requirements of grammatical structure, but ready to activate the final common path, if the effectiveness of this check is in any way interfered with" (1961, p. 186). But again, viewing the scanner as a device for serial disinhibition leads to the same predictions as the scanning mechanisms discussed just above: our data tolerate all three versions of the scanner.

Even so, the phenomenon of stress pre-entry presents major problems for other theories of serial order. For example, Conrad's (1965) proposal that serial-order errors are multiple substitutions occurring simply by chance provides no insight into stress preentry and is essentially the null hypothesis that our data refute.

Wickelgren's (1969) chain-association model of spoonerisms must also undergo serious revision to explain stress pre-entry. Chain association represents a radical departure from the mechanisms considered so far. In associative models, the motor units, rather than being independent of the serial-ordering mechanism, are linked by directional bonds, so that an entire word may be released in order by triggering the first motor unit. Associative models have difficulty explaining how sequences are continued after a serial-order reversal occurs. That is, if the correct sequence is $A B C D$ and the first two elements are reversed, present chainassociation models predict the continuation $B A B C D$ rather than the $B A C D$ that we find. This difficulty may be overcome if the links serve to boost the activation of the motor units to threshold and these units are viewed as variable resistors in the chain rather than all-or-none devices. Moreover, the only way I can see for 
associative models to handle stress pre-entry is to represent stress in the links between motor elements, rather than in the motor elements themselves. Further research to test these new assumptions is needed before chain association can be considered a viable principle in motor systems.

This résumé of alternative theories is by no means comprehensive but should perhaps suffice to refute Freud's assertion that "no matter what may be said about faulty actions, the whole subject is known to everybody as something self-evident" (1914, p. 114).

\section{Notes}

This work was supported by UCLA Grant 2426 and USPHS Grant 16668-01. The author thanks T. Falbo for running subjects and analyzing data, Professor R. C. Oldfield for providing space and equipment for the study at the M. R. C. Speech and Communication Unit, Edinburgh University, and Dr. G. Soderberg for running the control conditions. Received for publication June 19, 1970.

1. This was because analysis of stress, defined in terms of relative motor intensity, could be reliably determined only for syllables within a single word.

2. Comparison of syllabic stress in different words in a written corpus was out of the question, since different words may there be stressed to different extents. This 'word stress' would alter the relative motor intensity: of syllables in different words but not within a single word, and could only be determined from a tape recording of the error. Thus, analysis of stress in our corpus seemed justified only for within-word spoonerisms, not for betweenword spoonerisms.

3. Analysis of between-word spoonerisms showed a slightly different pattern of stress. Of the between-word reversals in Meringer's corpus 97\% occurred in syllables that were both stressed, an outcome exceeding chance expectation at the .01 level, chi-square test. But this finding in no way opposes a stress pre-entry hypothesis, since the second word may have received greater stress than the first as predicted in the hypothesis. We had no way of testing this question with our present data. Nevertheless, Boomer and Laver were able to analyze the role of word stress in a corpus of tape-recorded spoonerisms, and their analysis seems to support the stress pre-entry hypothesis: the second word in between-word spoonerisms was usually emphasized in their data, suggesting that "the tonic syllable is the only element with sufficient neurophysiological prominence to break through the inhibitory forces holding the programme in check" (1968).

4. Analysis of the between-word reversals in English showed the same pattern of stress as in German. In $88 \%$ of the corpus the syllables containing reversed phonemes were both stressed. Again, however, we had no way of analyzing the word stress superimposed on the syllabic stress, so that these data may also support the stress pre-entry hypothesis (see n. 3). 
5. An additional variable was manipulated in this and the next experiment. Half the subjects heard a tape recording of Strauss's "Thunder and Lightning Polka" while producing the sequences. This tended to increase the probability of all error types, but its detailed effect is left for a later report, since the effect on serial-order errors alone failed statistical significance.

6. That is, consonant reversals are most likely when similar consonants are preceded or followed by identical vowels (see MacKay, 1970).

7. Note that this view of stress would also explain why stressed syllables predominate in the partial recall of words (see Brown and McNeil, 1965).

\section{References}

Adrian, E. D., and Matthews, R. 1927. The action of light on the eye. Journal of Physiology 63:378-414.

Bawden, H. H. 1900. A study of lapses. Psychological Monographs 3(4) :1-121.

Boomer, D. S., and Laver, J. D. M. H. 1968. Slips of the tongue. British Journal of Disorders of Communication 3(1):1-12.

Brown, R., and McNeil, D. 1965. The tip of the tongue phenomenon. Journal of Verbal Learning and Verbal Behavior 3:325-327.

Bryden, M. P. 1967. A model for the sequential organization of behavior. Camadian Journal of Psychology 21(1):37-56.

Buller, A. J., Eccles, J. C., and Eccles, R. M. 1960. Interaction between motorneurons and muscles in respect of the characteristic speeds of their responses. Journal of Physiology 150:417-439.

Cohen, A. 1967. Errors of speech and their implication for understanding the strategy of speech users. Zeitschrift für Phonetik 20:177-181.

Conrad, R. 1965. Order errors in immediate recall of sequences. Journal of Verbal Learning and Verbal Behavior 4:161-169.

Freud, S. 1914. Psychopathology of everyday life, trans. A. Brill. New York: Penguin.

Heffner, R. M. S. 1964. General phonetics. Madison: University of Wisconsin Press.

Korte, A. 1915. Kinematoskopische Untersuchungen. Zeitschrift für Psychologie 72:123-126.

Lashley, K. S. 1961. The problem of serial order in behavior. In Psycholinguistics, ed. S. Saporta, pp. 180-198. New York: Holt, Rinehart and Winston.

Liang, T., and Piéron, H. 1942. Récherches sur la latence de la sensation lumineuse par la méthode de l'effet chromostéréoscopique. Année Psychologique 1:1-53.

Lit, A. 1949. The magnitude of the Pulfrich stereophenomenon as a function of binocular differences of intensity at various levels of illumination. American Journal of Psychology 62:159-181.

MacKay, D. G. 1969. Forward and backward masking in motor systems. Kybernetik $2: 57-64$. 
MacKay, D. G. 1970. Spoonerisms: The structure of errors in the serial order of speech. Neuropsychologia 8:323-350.

MacNeilage, P.F. 1964. Typing errors as clues to serial ordering mechanisms in language behavior. Language and Speech 7:155-159.

Meringer, R. 1908. Aus dem Leber der Sprache: Versprechen, Kindersprache, Nachahmungstrieb. Berlin: Behr's Verlag.

Meringer, R., and Mayer K. 1895. Versprechen und Verlesen: Eine psychologisch-linguistische Studie. Stuttgart: Göschensche Verlagsbuchhandlung.

Milner, P. M. 1961. A neural mechanism for the immediate recall of sequences. Kybernetik $1: 76-81$.

O'Hala, J. J. 1969. Aspects of the control and production of speech. Unpublished doctoral dissertation, University of California, Los Angeles.

Pulfrich, G. 1922. Die Stereoskopie im Dienste der isochromem und heterochromen Photometrie. Naturwissenschaften 10:533-761.

Shaffer, L. H., and Hardwick, J. 1968. Typing performance as a function of text. Quarterly Journal of Experimental Psychology 20:360-369.

Shaffer, L. H., and Hardwick, J. 1969. Errors and error detecting in typing. Quarterly Journal of Experimental Psychology $21: 209-213$.

Sutherland, N. S. 1959. Stimulus analyzing mechanisms. In Proceedings of the symposium on the mechanization of thought processes, vol. 2, pp. 575-609. London: H. M. S. O.

Wahrig, G., ed. 1966. Das grosse deutsche Wörterbuch. Munich: Bertelsmann Verlag.

Wickelgren, W. A. 1969. Context-sensitive coding, associative memory, and serial order in (speech) behavior. Psychological Review 76:1-15.

Wilson, J. A., and Anstis, S. M. 1969. Visual delay as a function of luminance. American Journal of Psychology 82:350-358.

Wundt, W. 1907. Outlines of psychology, trans. C. H. Judd. Leipzig: Wilhelm Engelmann. 